\title{
Imaging Patterns of Encephalopathy in Patients with COVID-19
}

\author{
Kumail Khandwala, Fatima Mubarak and Muhammad Nadeem Ahmad \\ Department of Radiology, The Aga Khan University Hospital, Karachi, Pakistan
}

\begin{abstract}
The aim of this retrospective observational study was to describe the neuroimaging manifestations of patients with COVID-19. This study was conducted at Aga Khan University Hospital, Karachi, Pakistan from March to July 2020. COVID-19 patients with neurological symptoms and positive neuroimaging were included after confirmation of COVID-19 by polymerase chain reaction test (PCR). In the 12 included patients, seizures and altered mentation were predominant neurological manifestations. Three cases had acute watershed infarcts (25\%), two cases had posterior cerebral artery territorial infarcts (16.7\%), two cases had periventricular corona radiata infarcts (16.7\%), three cases had hypoxic ischemic encephalopathy $(25 \%)$, two cases had posterior reversible encephalopathy syndrome (16.7\%), and there was one case each of cerebral venous sinus thrombosis, pontine infarct, and bithalamic lesions (8.3\%). This study highlights the diagnostic approaches in COVID-19-associated encephalopathy and the variable imaging features that clinicians and neuroradiologists should be aware of, as the pandemic progresses.
\end{abstract}

Key Words: COVID-19, Neuroimaging, Encephalopathy, Magnetic resonance imaging, Coronavirus.

How to cite this article: Khandwala K, Mubarak F, Ahmad MN. Imaging Patterns of Encephalopathy in Patients with COVID-19. J Coll Physicians Surg Pak 2021; 31(JCPSPCR):CR42-CR45.

Coronavirus disease (COVID-19) originated in December 2019 in Wuhan, China, probably from an animal source, and has since then had a rapid rate of transmission to the rest of the world, and being declared a global pandemic by the World Health Organisation. The typical symptoms of COVID-19 are respiratory-related. The elderly, especially those with comorbid conditions, are more likely to develop critical illness resulting in high mortality rates in this age group.

COVID-19 has characteristic laboratory findings and lung radiographic and computed tomography (CT) abnormalities. However, neuroimaging features in COVID-19 patients have not been widely documented asyet. According to the available literature, patients with COVID-19 infection can present with acute encephalopathy with changes in their level of consciousness, acute stroke, as a result of thromboembolic phenomena and/or seizures. ${ }^{1}$

In this study, the authors report cases of COVID-19 patients, who presented with neurological manifestations, and describe their relevant neuroimaging features. To date, these are the first reported cases of COVID-19 from the subcontinent with positive findings on neuroimaging.

Correspondence to: Dr. Fatima Mubarak, Department of Radiology, The Aga Khan University Hospital, Karachi, Pakistan

E-mail: mubarakfatima6@gmail.com

Received: July 05, 2020; Revised: November 13, 2020;

Accepted: December 10, 2020

DOI: https://doi.org/10.29271/jcpsp.2021.JCPSPCR.CR42
This was a retrospective-descriptive study commenced after approval was obtained from the Ethical Review Committee. Patients with COVID-19, who presented with neurological symptoms to the Aga Khan University Hospital, Karachi, Pakistan from March 2020 to July 2020, were included after they had neuroimaging done from the Radiology Department of this hospital. Severe acute respiratory syndrome coronavirus 2 (SARS-CoV-2) infection was confirmed by quantitative RT-PCR by nasopharyngeal or oropharyngeal swab. Patient demographic data, comorbid conditions, neurological symptoms and relevant investigations (laboratory and neurophysiology) were retrieved from the electronic records of the patients. Patients who had negative imaging findings were excluded.

Data entry and analyses were carried out on Microsoft Excel. Quantitative demographic data were reported as mean \pm standard deviation. The qualitative data was expressed as frequency (percentage).

A total of 12 patients with SARS-CoV-2 infection met the inclusion criteria and had neurological involvement with positive imaging findings. There were two pediatric patients. The mean age was $48.1 \pm 28.3$ years (range, $0.5-81$ ). A summary of the demographic data, clinical and neuroimaging features are provided in Table I. Neurological symptoms included seizures and altered mentation ( $n=5)$, drowsiness ( $n=4)$, encephalopathy $(n=3)$, acute loss of consciousness $(n=2)$, motor weakness $(n=3)$, and headache and anosmia $(n=1)$. Neurological symptoms were part of the initial presentation in four patients $(33.3 \%)$. 
Table I: Summary of patient demographic details, comorbidities, clinical presentation and neuroimaging findings.

\begin{tabular}{|c|c|c|}
\hline $\begin{array}{l}\text { Patient demographics \& } \\
\text { comorbidities }\end{array}$ & Neurological clinical presentation & Neuroimaging findings \\
\hline 6 month old male, VSD and PDA & GTC, moderate encephalopathy & $\begin{array}{l}\text { Bilateral acute internal watershed territory infarcts with PRES } \\
\text { and microhemorrhages }\end{array}$ \\
\hline 3 year old female, NKCM & GTC, altered mentation, quadriparesis & Bithalamic non-diffusion restricted lesions \\
\hline 29 year old male, NKCM & $\begin{array}{l}\text { Anosmia, GTC, headache, mild } \\
\text { encephalopathy }\end{array}$ & $\begin{array}{l}\text { Cerebral venous sinus thrombosis with hemorrhagic venous } \\
\text { infarct }\end{array}$ \\
\hline 35 year old male, NKCM & Acute loss of consciousness & Global hypoxic ischemic encephalopathy \\
\hline $\begin{array}{l}38 \text { year old female, SLE and } \\
\text { hypothyroidism }\end{array}$ & $\begin{array}{l}\text { GTC and altered mentation, severe } \\
\text { encephalopathy }\end{array}$ & $\begin{array}{l}\text { PRES, holohemispheric encephalopathy without diffusion } \\
\text { restriction }\end{array}$ \\
\hline 41 year old male, NKCM & Drowsiness, altered mentation & $\begin{array}{l}\text { Global hypoxic ischemic encephalopathy and multifocal } \\
\text { microhemorrhages }\end{array}$ \\
\hline 56 year old male, NKCM & GTC & Global hypoxic ischemic encephalopathy/postictal \\
\hline $\begin{array}{l}68 \text { year old female, DM, HTN and } \\
\text { asthma }\end{array}$ & Drowsiness and altered mentation & $\begin{array}{l}\text { Right posterior cerebral artery acute infarct with hemorrhagic } \\
\text { conversion }\end{array}$ \\
\hline 72 year old female, CLD & Acute loss of consciousness & Small acute infarct in left corona radiata \\
\hline 75-year old male, DM, CHD & $\begin{array}{l}\text { Drowsiness and new onset left sided } \\
\text { peripheral motor weakness }\end{array}$ & $\begin{array}{l}\text { Subacute infarction in the right posterior cerebral artery } \\
\text { territory with hemorrhagic conversion }\end{array}$ \\
\hline 79-year-old female, DM, HTN & Drowsiness and altered mentation & Bilateral internal watershed infarcts, pontine infarct \\
\hline $\begin{array}{l}81 \text { year old male, chronic atrial } \\
\text { fibrillation and asthma }\end{array}$ & $\begin{array}{l}\text { Drowsiness and new onset left sided } \\
\text { hemiparesis }\end{array}$ & $\begin{array}{l}\text { Left internal watershed infarcts, left occipital lobe, } \\
\text { periventricular corona radiata }\end{array}$ \\
\hline
\end{tabular}

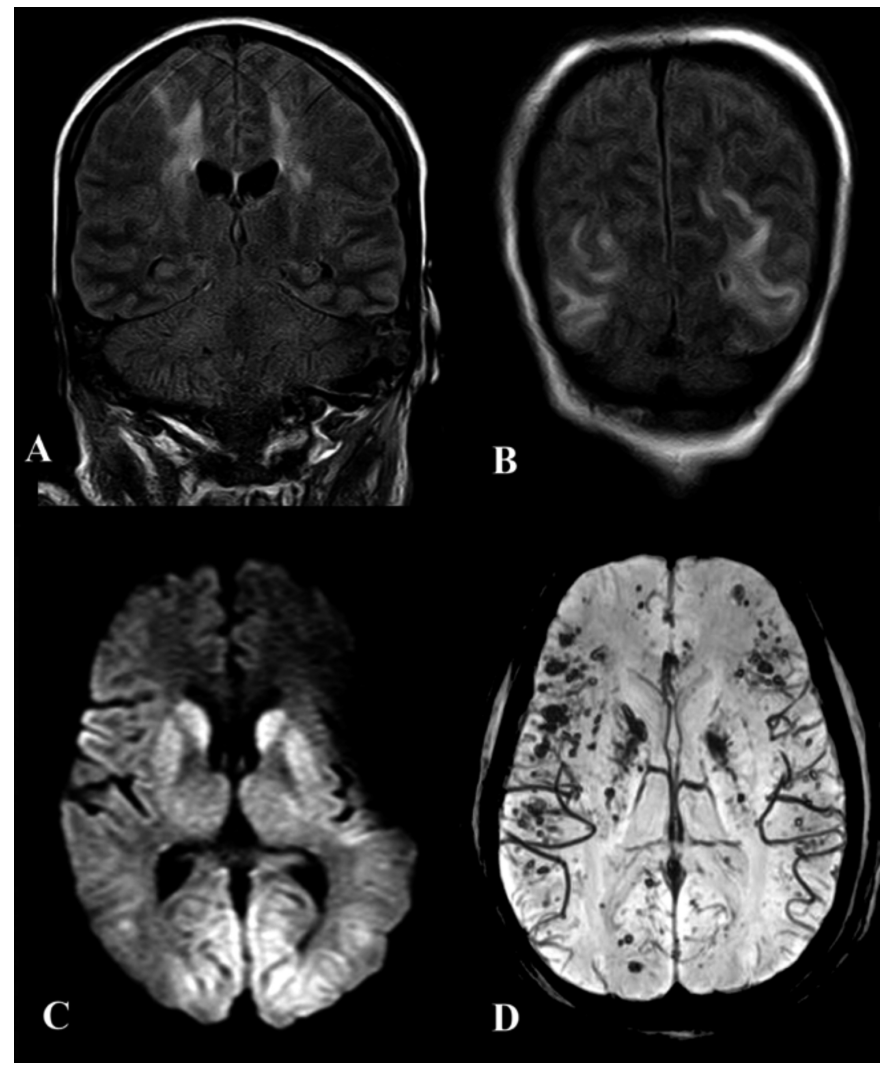

Figure 1: (A\&B) Coronal FLAIR images of a 38-year female, showing hyperintense signals in the parieto-occipital white matter and bilateral centrum semiovale, suggestive of PRES and a holohemispheric pattern of encephalopathy (arrows). These signals did not show diffusion restriction. (C) Axial DWI B-value 1000 image showing diffusion restriction in the basal ganglia and posterior parieto-occipital cortices of a $\mathbf{4 1}$ year old male suggestive of hypoxic ischemic injury. (D) Multifocal susceptibility dropout in the same patient, indicative of microhemorrhages.

Systemic manifestations included fever $(n=7)$, dyspnea $(n=4)$, cough $(n=3)$, and hypernatremic dehydration $(n=$ 1 ). Five patients $(41.7 \%)$ had no known comorbidities. Electroencephalography showed mild, moderate and severe encephalopathy in the three out of four patients tested. One patient had continuous epileptiform discharges with myoclonic jerks and was subsequently diagnosed with postictal encephalopathy on imaging. Eight patients (66.7\%) showed raised $D$-dimer levels (above the normal reference values).

Neuroimaging revealed three cases with acute diffusion restricted internal border zone watershed infarcts (25\%), and two cases with acute and subacute right sided posterior cerebral artery territorial infarcts with areas of hemorrhagic conversion (16.7\%). There were three cases of hypoxic ischemic encephalopathy (25\%), two cases of posterior reversible encephalopathy syndrome (16.7\%), two cases of 
multiple microhemorrhages (16.7\%) two cases of periventricular corona radiata infarcts $(16.7 \%)$, and one case each of cerebral venous sinus thrombosis, pontine infarct, and nondiffusion restricted bi-thalamic lesions (8.3\%, Figures 1 and 2 ).

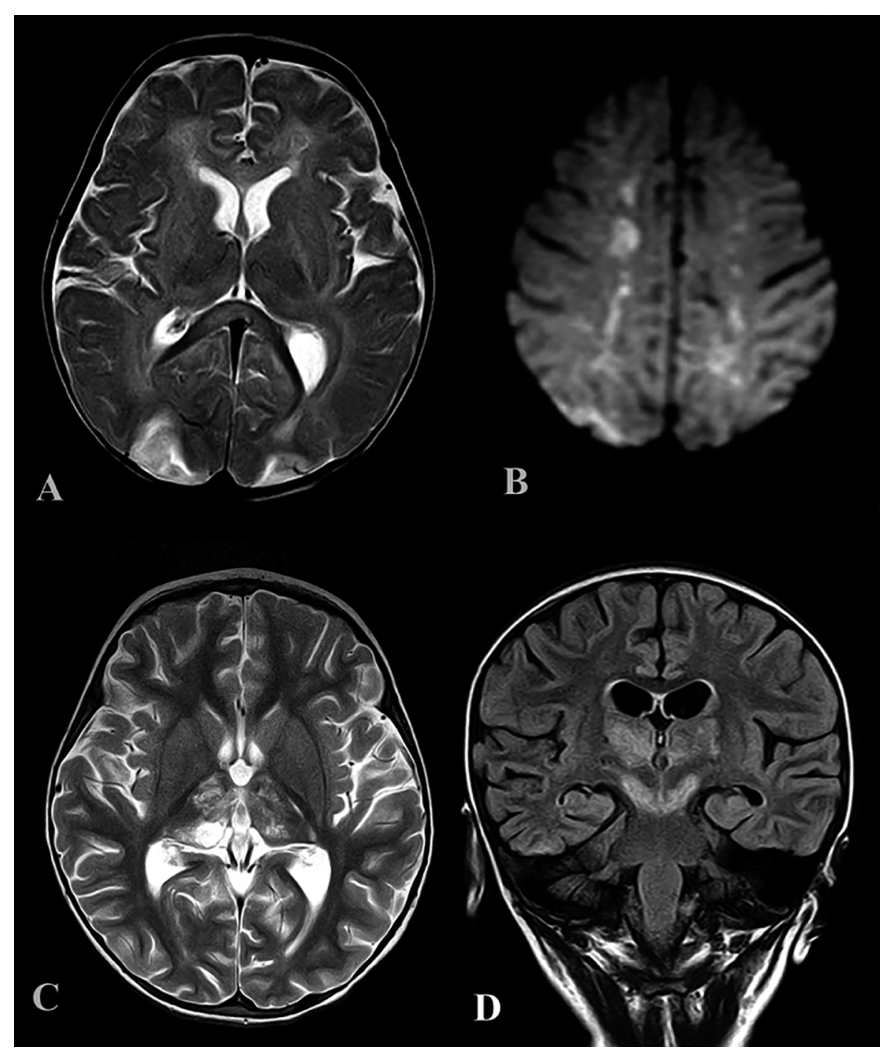

Figure 2: (A) Axial T2 weighted sequence showing bilateral parieto-occipital hyperintensities, suggestive of PRES, with splenial signal abnormality in a 6-month boy with COVID-19. (B) Axial DWI in the same patient showing bilateral diffusion restricted internal watershed territory infarcts. (C\&D) Axial T2 and coronal FLAIR in a 3-year girl with bithalamic signal abnormalities extending to the cerebral peduncles. These were not diffusion restricted or showing any hemorrhage.

SARS-COV-2 may enter the central nervous system (CNS) through the hematogenous or retrograde neuronal route, as seen with other respiratory viruses. The latter is advocated by the fact that some patients had experienced anosmia, including one patient in this series. The data regarding COVID-19 neurological manifestations and neuroimaging features is scarce. ${ }^{1}$ This study demonstrated that the neuroimaging features of COVID-19 patients were variable. Acute ischemic infarcts and different patterns of encephalopathy were predominant in these patients. The encephalopathy spectrum in this series included posterior reversible encephalopathy syndrome (PRES), global hypoxic-ischemic encephalopathy, and cortical and deep grey matter pattern of T2 FLAIR hyperintense signal with associated restriction diffusion that may be caused by hypoxic/ischemic or postictal effects. This has also been similarly reported by Mahammedi et al. ${ }^{2}$

Bilateral frontotemporal hypoperfusion has been noted in patients who underwent perfusion imaging in a prior study. ${ }^{3}$
It was, however, found that the patterns of encephalopathy encountered predominantly involving the posterior circulation and watershed areas, suggesting that these areas are prone to hypoperfusion and are therefore more vulnerable in this disease. Both cases of acute territorial infarction in this study involved the posterior cerebral artery territory, with areas of hemorrhagic conversions, also indicating that these are the more likely arterial territories to be affected. A plausible explanation may be deficient adrenergic sympathetic innervations in the vertebrobasilar system than in the carotid system. A proposition for increased incidence of stroke and thromboembolic phenomena in COVID-19 may also be cytokine storm syndrome, related to the prothrombotic effect of the inflammatory response. ${ }^{4}$

Two patients had multifocal microhemorrhages; one patient with extensive cerebral venous sinus thrombosis involving the superior sagittal sinus, right transverse and sigmoid sinuses had a large hemorrhagic venous infarct in the right frontal lobe. There was one patient with spontaneous intracerebral hemorrhage and cerebral venous sinus thrombosis each in the study from Wuhan, China, as well. ${ }^{1}$ It has been hypothesised that brain angiotensin converting enzyme II could be involved in COVID-19 infection; and its dysfunction, leading to disruption of cerebral autoregulation and hemorrhage, due to arterial wall rupture. ${ }^{5}$

This study also included two pediatric cases, one of whom had watershed ischemic insults with PRES and microhemorrhages, and the other had bithalamic non-diffusion restricted lesions without any hemorrhage or enhancement. Interestingly, the first reported case of COVID-19-associated acute necrotising hemorrhagic encephalopathy showed involvement of bilateral thalami. These types of bithalamic abnormalities have also been noted in other viral encephalitides. Signal abnormality was seen in the splenium of corpus callosum of the pediatric patient with watershed infarcts and PRES. This is similar to a recent study in which acute splenial lesions were noted in all four pediatric cases with COVID-19 related encephalopathies. ${ }^{6}$ The changes are thought to be attributable to focal intramyelinic edema secondary to inflammation, but may also be seen in other types of viral illnesses.

Regarding meningoencephalitis, none of our cases showed abnormal parenchymal or leptomeningeal enhancement. A study done in France showed a high incidence of leptomeningeal enhancement, predominantly in occipital lobe in 8 out of their 13 patients. ${ }^{3}$ Some authors, however, propose that CSF titers of the virus may be extremely low and dissemination is usually transient.

The key limitations of this study are the small sample size and single-centre experience. Additionally, it could not be determined whether these findings were coincidental or specific to COVID-19 infection. Further studies are warranted to validate the pathogenesis and mechanism of neurological 
involvement in this multisystemic viral syndrome. However, neurologists and neuroradiologists should be aware of the wide spectrum of neuroimaging patterns associated with COVID-19, as this pandemic progresses. Overall, this article indicates that the CNS may be variably involved in COVID-19 and highlights the diagnostic approaches to SARS-CoV-2 associated encephalopathy.

\section{CONFLICT OF INTEREST:}

Authors declared no conflict of interest.

\section{AUTHORS' CONTRIBUTION:}

KK: Manuscript writing, literature review, data collection and analysis.

FM: Conceptualization of study, literature review and data interpretation.

MNA: Data interpretation, design of work and critical review. All authors contributed towards critical review and final approval of the manuscript.

\section{REFERENCES}

1. Mao L, Wang M, Chen S, He Q, Chang J, Hong C, et al. Neurological manifestations of hospitalised patients with
COVID-19 in Wuhan, China: A retrospective case series study. JAMA Neurol 2020; 77(6):683-90. doi:10.1001/jamaneurol.2020.1127.

2. Mahammedi A, Saba L, Vagal A, Leali M, Rossi A, Gaskill M, et al. Imaging in neurological disease of hospitalised COVID-19 patients: An Italian multicenter retrospective observational study. Radiology 2020; 297(2):E270-E3. doi:10.1148/radiol.2020201933.

3. Helms J, Kremer S, Merdji H, Clere-Jehl R, Schenck M, Kummerlen $C$, et al. Neurologic features in severe SARS-CoV-2 infection. N Engl J Med 2020; 382(23):2268-70. doi:10.1056/NEJMc2008597.

4. Mehta P, McAuley DF, Brown M, Sanchez E, Tattersall RS, Manson JJ. COVID-19: consider cytokine storm syndromes and immunosuppression. Lancet 2020; 395(10229): 1033-4. doi: 10.1016/S0140-6736(20)30628-0.

5. Sharifi-Razavi A, Karimi N, Rouhani N. COVID-19 and intracerebral haemorrhage: Causative or coincidental? New Microbes New Infect 2020; 35:100669. doi 10.1016/j.nmni.2020.100669.

6. Abdel-Mannan O, Eyre M, Löbel U, Bamford A, Eltze C, Hameed $B$, et al. Neurologic and radiographic findings associated with COVID-19 infection in children. JAMA Neurol 2020; 77(11):1-6. doi:10.1001/jamaneurol.2020.2687. 